\title{
Application of Thermodynamic Analysis in Reducing Detailed Hydrogen Combustion Mechanism
}

\author{
V.G. Matveev ${ }^{1}$, A.A. Molokanov ${ }^{2,3}$, L.S. Yanovskiy ${ }^{2,3,4}$ \\ ${ }^{1}$ LLC "Fireworks", Chernogolovka, Moscow Region, 142432, Russia, \\ ${ }^{2}$ Central Institute of Aviation Motors, Moscow, 111116, Russia, \\ ${ }^{3}$ Institute of Problems of Chemical Physics of Russian Academy of Sciences, \\ Chernogolovka, Moscow Region, 142432, Russia \\ ${ }^{4}$ Moscow State University, Moscow, 119991, Russia \\ vgmatv@mail.ru,molokanov@ciam.ru,yanovskiy@ciam.ru
}

\begin{abstract}
A set of programs has been created; it allows to carry out the thermodynamic analysis and kinetic computation of complex chemical reactions. A minimum mechanism describing the combustion kinetics of hydrogen is determined; the mechanism was used to solve an inverse task of finding parameters describing the experimental data of Kowalski at pressures of 7.4, 7.1, 6.8, 6.4 and $6.1 \mathrm{~mm} \mathrm{Hg}$. All obtained constants of direct and inverse reactions are interrelated by thermodynamic equilibrium constants. The parameters obtained for the maximum hydrogen combustion mechanism make it possible to describe well the ignition limits in Lewis and Egerton experiments. In carrying out further thermodynamic analysis, a minimal mechanism M-I is identified that corresponds to the maximum mechanism and with good accuracy describing the critical conditions of hydrogen combustion in the pressure interval $1 \div 200 \mathrm{~mm} \mathrm{Hg}$ and temperatures of $400^{\circ} \mathrm{C} \div 600{ }^{\circ} \mathrm{C}$. From the analysis of critical conditions, an analytical equation is obtained; roots of the equation give ignition limits close to the experimental ones.
\end{abstract}

Keywords: hydrogen combustion kinetic, thermodynamic analysis, reduction of kinetic mechanism.

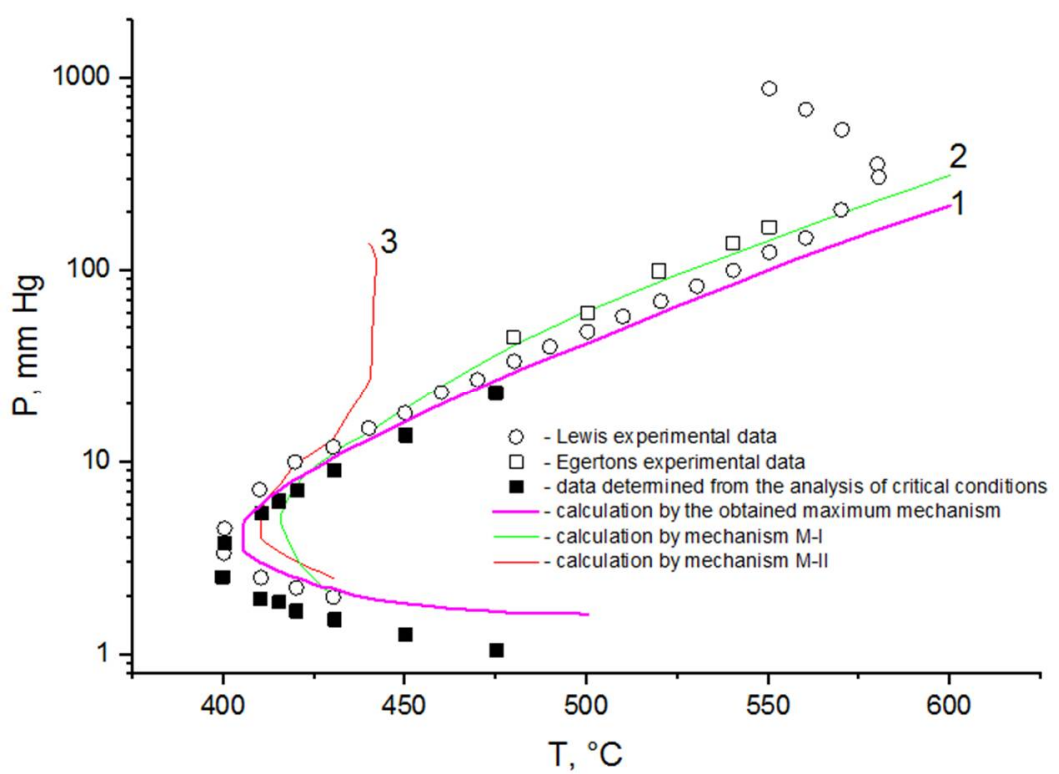

Comparison of calculated and experimental data of ignition limits of stoichiometric mixture of $\mathrm{H}_{2}$ and $\mathrm{O}_{2}$ 


\title{
Применение метода термодинамического анализа при сокращении размерности детального механизма горения водорода
}

\author{
В.Г. Матвеев ${ }^{1}$, А.А. Молоканов ${ }^{2,3}$, Л.С. Яновский ${ }^{2,3,4}$ \\ ${ }^{1}$ ООО «Фейерверк», Россия, г. Черноголовка МО, 142432, \\ пр-т ак. Семенова, 1, корп. 1/8, оф. 4 \\ ${ }^{2}$ ФГУП «ЦИАМ им. П.И. Баранова», Россия, Москва, 111116, ул. Авиамоторная, 2 \\ ${ }^{3}$ Институт проблем химической физики РАН, Россия, г. Черноголовка МО, 142432, \\ пр-т ак. Семенова, 1 \\ ${ }^{4}$ МГУ имени М.В.Ломоносова, Россия, Москва, 119991, Ленинские горы, д. 1 \\ vgmatv@mail.ru,molokanov@ciam.ru, yanovskiy@ciam.ru
}

\begin{abstract}
Аннотация
Создан комплекс программ, позволяющий проводить термодинамический анализ и выполнять кинетические расчеты сложных химических реакций. Определена минимальная схема, достаточная для описания кинетики горения водорода, которая была использована при решении обратной задачи и поиска набора параметров, описывающих экспериментальные данные Ковальского при давлениях 7.4, 7.1, 6.8, 6.4 и 6.1 мм.рт.ст. Все полученные константы прямых и обратных реакций взаимосвязаны термодинамическими константами равновесия. Полученные параметры для максимальной схемы горения водорода позволяют хорошо описать пределы воспламенения в экспериментах Льюиса и Эгертона. При проведении дальнейшего термодинамического анализа выделен минимальный механизм, соответствующий максимальному и с хорошей точностью описывающий критические условия горения водорода в интервале давлений $1 \div 200$ мм.рт.ст. и температур $400{ }^{\circ} \mathrm{C} \div 600{ }^{\circ} \mathrm{C}$. Из анализа критических условий получено аналитическое уравнение, корни которого дают пределы воспламенения близкие к экспериментальным.
\end{abstract}

Ключевые слова: кинетика горения водорода, термодинамический анализ, редуцирование кинетической схемы.

\section{1. Введение}

В последнее время вследствие развития альтернативной энергетики возрастает интерес к моделированию процессов горения водорода при разработке перспективных авиационных двигателей [1]. Однако при решении задач гидродинамики, распространения пламени или детонационных волн учет всех элементарных стадий кинетики химической реакции горения водорода не всегда возможен [2-5]. Для адекватного описания таких процессов необходимо уметь выделять упрощенный механизм, в целом соответствующий реальным химическим реакциям и позволяющий описывать основные физико-химические закономерности [6-8].

Для редуцирования детальных механизмов предложено несколько способов. Так, в одном из подходов определяют относительную значимость реакций, используя величины

$$
\frac{\mathrm{d} y_{i}}{\mathrm{~d} y_{j}} \text { или } \frac{\mathrm{d} \ln \left(y_{i}\right)}{\mathrm{d} \ln \left(y_{j}\right)},
$$

характеризующие чувствительность концентрации $i$-го компонента к неопределенности константы скорости $k_{j} j$-й реакции, для быстрого вычисления которых предложены метод 
нелинейного Фурье-анализа [9] и метод функций Грина [10]. Однако из-за высокой сложности и низкой эффективности этот подход не получил широкого применения.

В другом методе [11] величина, называемая кинетической долей реакции $\alpha_{i j}$, представляет собой вклад $j$-й элементарной стадии в совокупности всех реакций, в которых участвует $i$-я компонента

$$
\alpha_{i j}=\frac{\gamma_{i j} \varpi_{j}}{\sum_{j} \gamma_{i j} \varpi_{j}},
$$

где $\gamma_{i j}$-стехиометрическая матрица; $\varpi_{j}-$ скорость $j$-й реакции. Однако получаемая характеристика является двумерной и не всегда однозначно отражает роль реакции.

В работе [8] предложен метод сокращения размерности кинетической схемы, основанный на выделении многообразия медленного изменения некоторых комбинаций переменных, соответствующих собственным значениям матрицы Якоби кинетической схемы с наименьшими отрицательными действительными частями. В частности, выделяются все линейные многообразия, соответствующие нулевым собственным значениям, т.е. законам сохранения.

Тем не менее, в литературе отсутствуют примеры последовательного применения какого-либо метода редуцирования кинетической схемы горения водорода и сравнения с экспериментальными данными в различных условиях.

В настоящей работе поставлена задача: из максимального механизма горения водорода выделить упрощенный механизм, состоящий из как можно меньшего числа реакций, но достаточно хорошо описывающий экспериментальные данные. В качестве экспериментальных данных были выбраны кинетический эксперимент Ковальского [12], результаты определения пределов воспламенения Льюиса [13] и Эгертона [14].

\section{2. Применение метода термодинамического анализа для редуцирования кинетической схемы горения водорода}

\section{1. Расчет пределов горения водорода по максимальному механизму}

Для кинетических расчетов и определения критических условий в данной работе был написан оригинальный пакет программ. Все расчеты были выполнены для изотермических условий.

Реакция окисления водорода исследовалась как экспериментально, так и теоретически $[13,15]$, максимальный механизм, который определяется в случае системы «водород - кислород» для набора частиц $\mathrm{H}_{2}, \mathrm{O}_{2}, \mathrm{OH}, \mathrm{H}, \mathrm{O}, \mathrm{HO}_{2}, \mathrm{H}_{2} \mathrm{O}, \mathrm{H}_{2} \mathrm{O}_{2}$ как механизм, состоящий из всех возможных линейно независимых реакций, включает в себя 30 обратимых реакций [16].

При использовании максимальной схемы горения водорода (30 обратимых реакций +4 реакции взаимодействия радикалов со стенкой) по данным [16] получены верхние и нижние значения пределов воспламенения. Сравнение полученных результатов с экспериментами данными Льюиса [13] и Эгертона [14] приведено на рис. 1. Наблюдаемый разброс пределов воспламенения, получаемых расчетным путем с использованием максимального механизма, обусловлен невысокой точностью определения параметров элементарных реакций максимального механизма. Использование максимального механизма [16] не позволяет точно описать экспериментальные данные $[13,14]$ и требует большого объема вычислительных мощностей, что еще раз подчеркивает актуальность настоящей работы. 


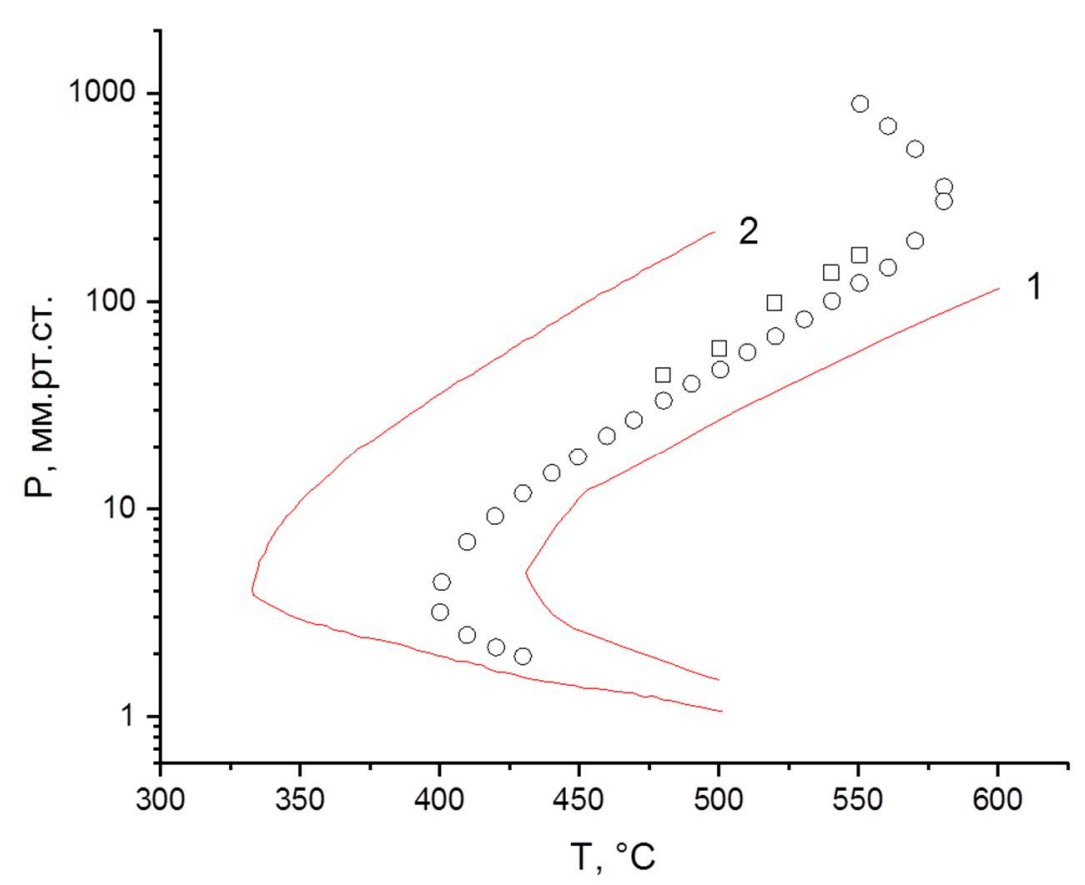

Рис. 1. Пределы воспламенения стехиометрической смеси $\mathrm{H}_{2}$ и $\mathrm{O}_{2}$.

Расчет по максимальному механизму [16]: кривая 1 - использованы верхние значения констант, кривая 2 - нижние.

o - экспериментальные данные [13], в - экспериментальные данные [14]

\section{2. Определение термодинамической доли элементарных реакций}

Применение метода термодинамического анализа $[17,18]$ позволяет оценить термодинамическую долю каждой элементарной стадии из максимального механизма горения водорода с кислородом. Для этого используется формула

$$
q_{j}=\frac{\left(\varpi_{j}^{+}-\varpi_{j}^{-}\right) \ln \left(\frac{\varpi_{j}^{+}}{\varpi_{j}^{-}}\right)}{\sum_{j}\left(\varpi_{j}^{+}-\varpi_{j}^{-}\right) \ln \left(\frac{\varpi_{j}^{+}}{\varpi_{j}^{-}}\right)},
$$

где $q_{j}$ - неравновесная термодинамическая характеристика, термодинамическая доля $j$-й стадии; $j$-я стадия представляет собой совокупность прямой и обратной реакций, скорости которых $\varpi_{j}^{+}$и $\varpi_{j}^{\bar{j}}$, соответственно.

Для отбора элементарных реакций эта величина оказалась более представительной, чем упомянутые вначале статьи, поскольку она учитывает, как термодинамический фактор $\ln \left(\varpi_{j}^{+} / \varpi_{j}^{-}\right)$, определяющий отклонение реагирующей системы от равновесия, так и кинетический фактор $\left(\varpi_{j}^{+}-\varpi_{j}^{-}\right)$, характеризующий скорость приближения к равновесию.

Вычисленные термодинамические доли всех реакций как функции времени для максимального механизма позволяют сделать заключение о роли какой-либо реакции в отдельные моменты времени. Рассмотрим реакции 1, 18, 22 и 23 вместе с 9 (из максимальной схемы [16]), которые могут рассматриваться как инициирующие. Для условий эксперимента [12] расчет термодинамических долей элементарных реакций и кинетики горения стехиометрической смеси кислорода и водорода при температуре $430^{\circ} \mathrm{C}$ и 5 мм.рт.ст. и показал, что реакция 1 , безусловно, играет большую роль в начале процесса и является основной инициирующей. Значение реакции 18 несущественно вначале, но к концу периода индукции возрастает 
и становится важным (рис. 2). Реакция 22 во все моменты времени имеет очень маленькую долю и несущественна. Необходимо отметить, что роль реакции 23 с увеличением давления растет, но все равно она существенна только на самых ранних стадиях.

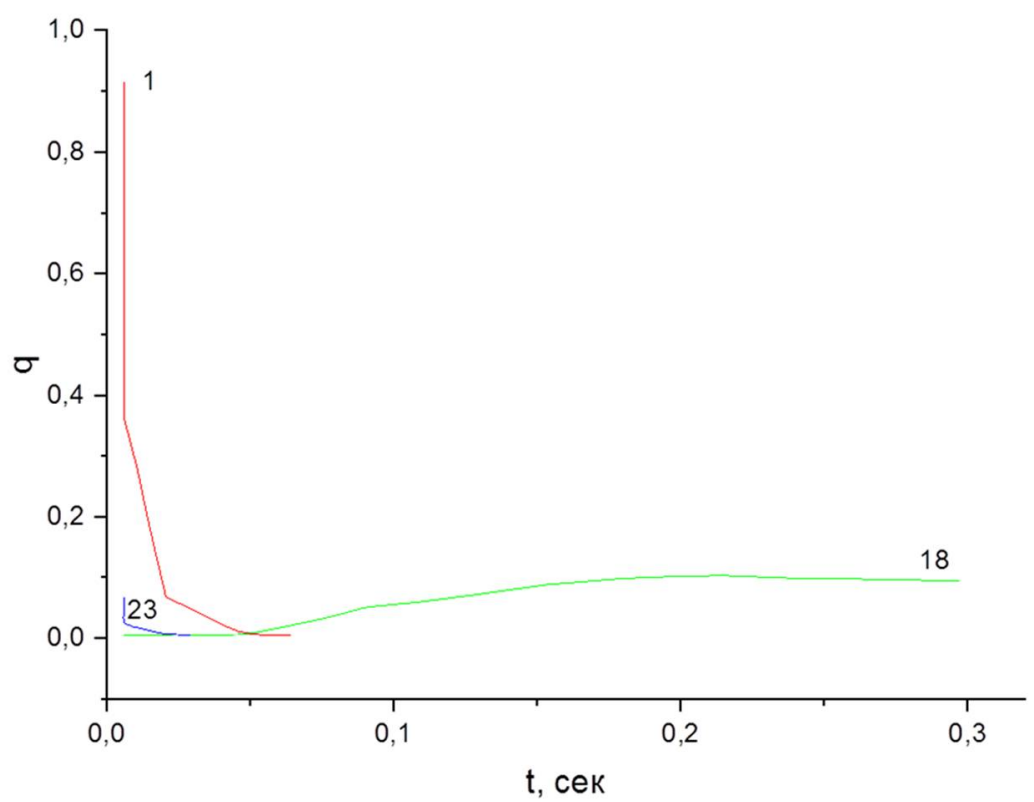

Рис. 2. Термодинамические доли реакций. Стехиометрическая смесь, $430{ }^{\circ} \mathrm{C}, 5$ мм.рт.ст.

Далее методом термодинамического анализа из максимального механизма [16] выбраны реакции, доля которых в некотором интервале времени составляет не менее 0.4 , в результате чего кинетическая схема сократилась до 8 реакций (номера 1, 2, 3, 4, 8, 11, 16, 32 по данным [16]).

\section{3. Поиск параметров элементрарных реакций горения водорода}

Полученная схема использована в обратной задаче поиска параметров с применением метода наискорейшего спуска [19] по экспериментальным данным [12], описывающим кинетические кривые горения стехиометрической смеси $\mathrm{H}_{2}$ и $\mathrm{O}_{2}$ при общем давлении 7.4, 7.1, 6.8, 6.4 и 6.1 мм.рт.ст. и температуре $T=485^{\circ}$ C. Необходимые для вычисления градиентов производные концентраций по параметрам получались при совместном решении системы дифференциальных уравнений для давление исходных веществ $\left(\mathrm{H}_{2}, \mathrm{O}_{2}\right)$, продуктов $\left(\mathrm{OH}, \mathrm{H}, \mathrm{HO}_{2}\right)$ и производных этих переменных по константам прямых реакций 1, 2, 3, 4, 8, 11, 16, 32. Система состояла из 48 уравнений и решалась методом Рунге-Кутта. По концентрациям О и $\mathrm{HO}_{2}$ предполагалось выполнение квазистационарности. Константы обратных реакций вычислялись по термодинамическим константам равновесия [19].

Поиск производился в направлении сближения констант, чтобы описание 5-ти выбранных экспериментальных кривых [12] осуществлялось одним набором параметров. После многократного прохождения по всем указанным параметрам и всем кинетическим кривым получено хорошее описание для всех давлений.

Особое внимание в работе было уделено гетерогенным реакциям на стенке [20, 21]. В данной работе принята простейшая модель гетерогенных реакций, и для вычисления констант скорости использовалась формула Семенова [20] для сферического сосуда

$$
k_{g}=\frac{4 \pi}{d^{2}} D\left(\frac{T}{T_{0}}\right)^{1.75} \frac{P_{0}}{P},
$$


где $d$ - диаметр сосуда; $D$ - коэффициент диффузии, равный для $\mathrm{OH}-0.34, \mathrm{H}-1.43, \mathrm{O}$ - 0.36 , $\mathrm{HO}_{2}-0.198 ; T, P$ - температура и давление в эксперименте; $T_{0}, P_{0}$ - соответственно, для нормальных условий.

В условиях эксперимента [12] формула (3) дает $k_{32}^{+}=211$. Это в 20 раз больше, чем получено при решении обратной задачи. Поэтому во всех расчетах по определению пределов воспламенения константы гетерогенных реакций вычислялись с этим коэффициентом.

Таким образом были уточнены параметры для 8 реакций, описывающих кинетику горения водорода. Все уточненные (полученные) константы прямых и обратных реакций согласованны с термодинамическими константами равновесия. При подстановке уточненных параметров 8 реакций горения водорода в максимальную схему, получили уточненную максимальную схему горения водорода [16], которая дает хорошее описание экспериментальных данных [12] (рис. 3).

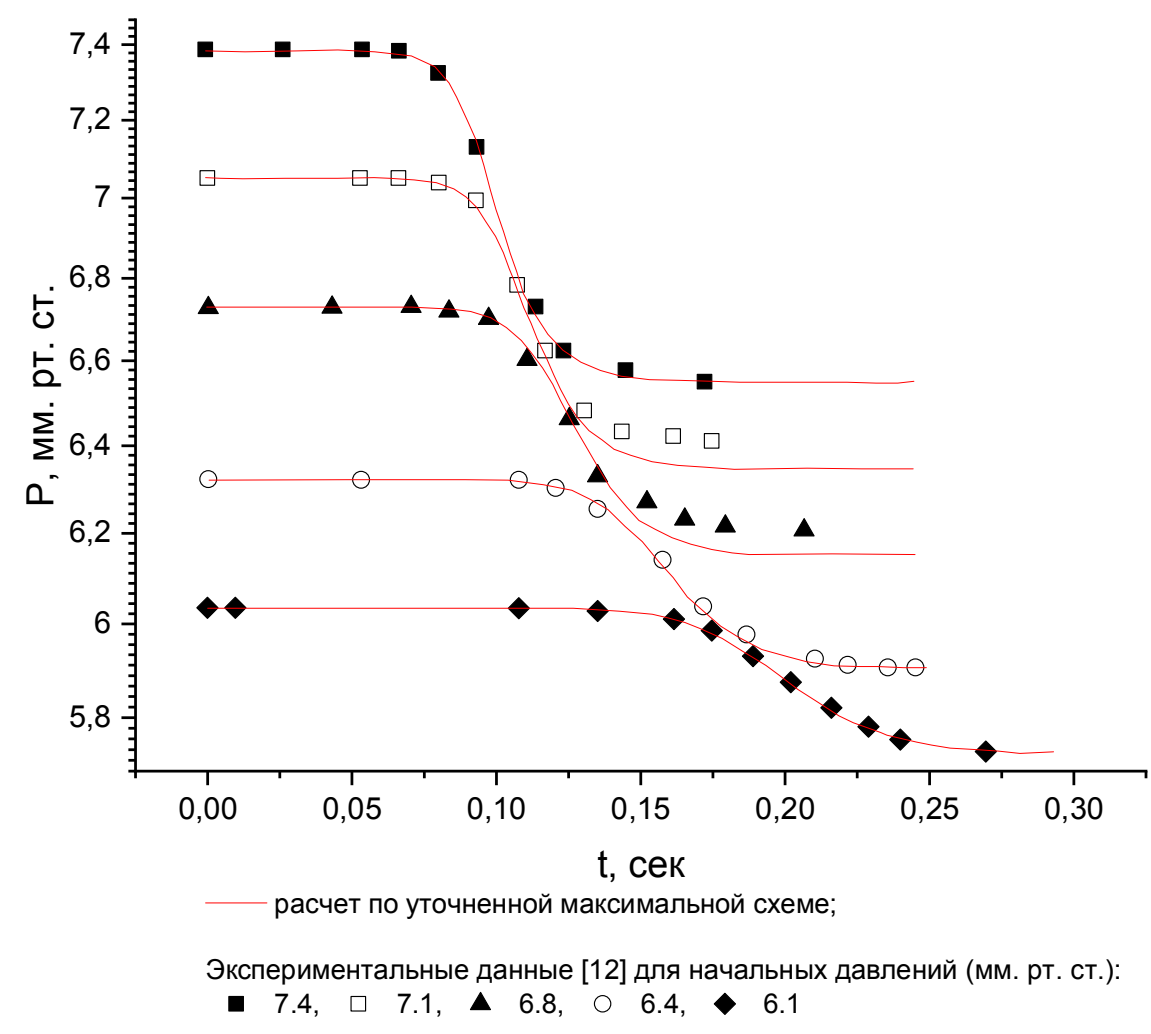

Рис. 3. Кинетические кривые горения стехиометрической кислород-водородной смеси

\section{4. Редуцирование полной кинетической схемы с уточненными параметрами}

Для полученной уточненной максимальной схемы, включающей 34 обратимых стадий, определили термодинамические доли $q_{j}$ отдельных стадий. Исключая все реакции, доля которых в момент времени от начала реакции до достижения равновесного состояния составляет менее 0.01 , был получен укороченный механизм, состоящий из 12 обратимых стадий МI - 1, 2, 3, 4, 8, 11, 16, 18, 31, 32, 33, 34 (табл. 1).

Исключение реакций 18 и 33, термодинамические доли которых самые маленькие среди оставшихся, приводит к механизму М-II.

На рис. 4 представлены кинетические кривые для концентраций $\mathrm{H}_{2}$ и $\mathrm{OH}$, рассчитанные по укороченным механизмам M-I и М-II в сравнении с расчетом по уточненному максимальному механизму. Видно, что переход от максимального к механизму М-І практически не меняет период индукции, тогда как для механизма М-II период индукции увеличивается на 0.1 сек, т.е. на $20 \%$, по сравнению с максимальным. 
Таблийа 1

Кинетическая схема механизма М-1 и ее параметры

\begin{tabular}{|c|c|c|c|c|c|c|c|}
\hline $\begin{array}{c}\text { № по } \\
{[16]}\end{array}$ & $\mathrm{A}^{+}$ & $\mathrm{N}^{+}$ & $\mathrm{E}^{+}$ & $\mathrm{A}^{-}$ & $\mathrm{n}^{-}$ & $\mathrm{E}^{-}$ & Реакция \\
\hline $1^{*}$ & $2.81 \mathrm{e} 10$ & 0. & 38.90 & $6.24 \mathrm{e} 08$ & 0. & 20.38 & $\mathrm{H}_{2}+\mathrm{O}_{2} \Leftrightarrow 2 \mathrm{OH}$ \\
\hline $2^{*}$ & $8.65 \mathrm{e} 10$ & 0. & $5 . \div 5.4$ & $3.21 \mathrm{e} 11$ & 0. & 20.82 & $\mathrm{H}_{2}+\mathrm{OH} \Leftrightarrow \mathrm{H}_{2} \mathrm{O}+\mathrm{H}$ \\
\hline $3^{*}$ & $4.42 \mathrm{e} 11$ & 0. & 17.60 & $2.20 \mathrm{e} 10$ & 0. & 0.98 & $\mathrm{O}_{2}+\mathrm{H} \Leftrightarrow \mathrm{OH}+\mathrm{O}$ \\
\hline $4^{*}$ & $7.07 \mathrm{e} 07$ & 1. & 8.95 & $3.15 \mathrm{e} 08$ & 1. & 7.05 & $\mathrm{H}_{2}+\mathrm{O} \Leftrightarrow \mathrm{OH}+\mathrm{H}$ \\
\hline $8^{*}$ & $1.27 \mathrm{e} 16$ & -2. & 0.00 & $1.81 \mathrm{e} 16$ & 0. & 118.20 & $\mathrm{H}+\mathrm{OH}+\mathrm{M} \Leftrightarrow \mathrm{H}_{2} \mathrm{O}+\mathrm{M}$ \\
\hline $11^{*}$ & $3.78 \mathrm{e} 09$ & 0. & -1.89 & $2.48 \mathrm{e} 14$ & 0. & 48.30 & $\mathrm{H}^{+} \mathrm{O}_{2}+\mathrm{M} \Leftrightarrow \mathrm{HO}_{2}+\mathrm{M}$ \\
\hline $16^{*}$ & $8.90 \mathrm{e} 09$ & 0. & 2.58 & $4.28 \mathrm{e} 08$ & 0. & 37.13 & $\mathrm{H}+\mathrm{HO}_{2} \Leftrightarrow \mathrm{OH}_{+} \mathrm{OH}$ \\
\hline 18 & $5.00 \mathrm{e} 09$ & 0. & 1.20 & $1.08 \mathrm{e} 10$ & 0. & 54.27 & $\mathrm{H}+\mathrm{HO}_{2} \Leftrightarrow \mathrm{H}_{2}+\mathrm{O}_{2}$ \\
\hline 31 & $3.04 \mathrm{e} 01$ & 0. & 0.00 & $1.0 \mathrm{e}-10$ & 0. & 0.0 & $\mathrm{OH}_{2}$ стенка $\Leftrightarrow \mathrm{OH}_{\mathrm{s}}$ \\
\hline $32^{*}$ & $1.05 \mathrm{e} 01$ & 0. & 0.00 & $1.0 \mathrm{e}-10$ & 0. & 0.0 & $\mathrm{H}+$ стенка $\Leftrightarrow \mathrm{H}_{\mathrm{s}}$ \\
\hline 33 & $2.95 \mathrm{e} 01$ & 0. & 0.00 & $1.0 \mathrm{e}-10$ & 0. & 0.0 & $\mathrm{O}+$ стенка $\Leftrightarrow \mathrm{O}_{\mathrm{s}}$ \\
\hline 34 & $4.64 \mathrm{e} 01$ & 0. & 0.00 & $1.0 \mathrm{e}-10$ & 0. & 0.0 & $\mathrm{HO}_{2}+$ стенка $\Leftrightarrow \mathrm{HO}_{2 \mathrm{~s}}$ \\
\hline
\end{tabular}

Примечание: А - предэкспонент в л/моль/сек для бимолекулярных и л²/моль $/$ сек тримолекулярных реакций; $\mathrm{n}$ - показатель степени температуры; $\mathrm{E}$ - энергия активации в ккал/моль + для прямой и для обратной реакций; * - стадии, параметры которых уточнены в настоящей работе

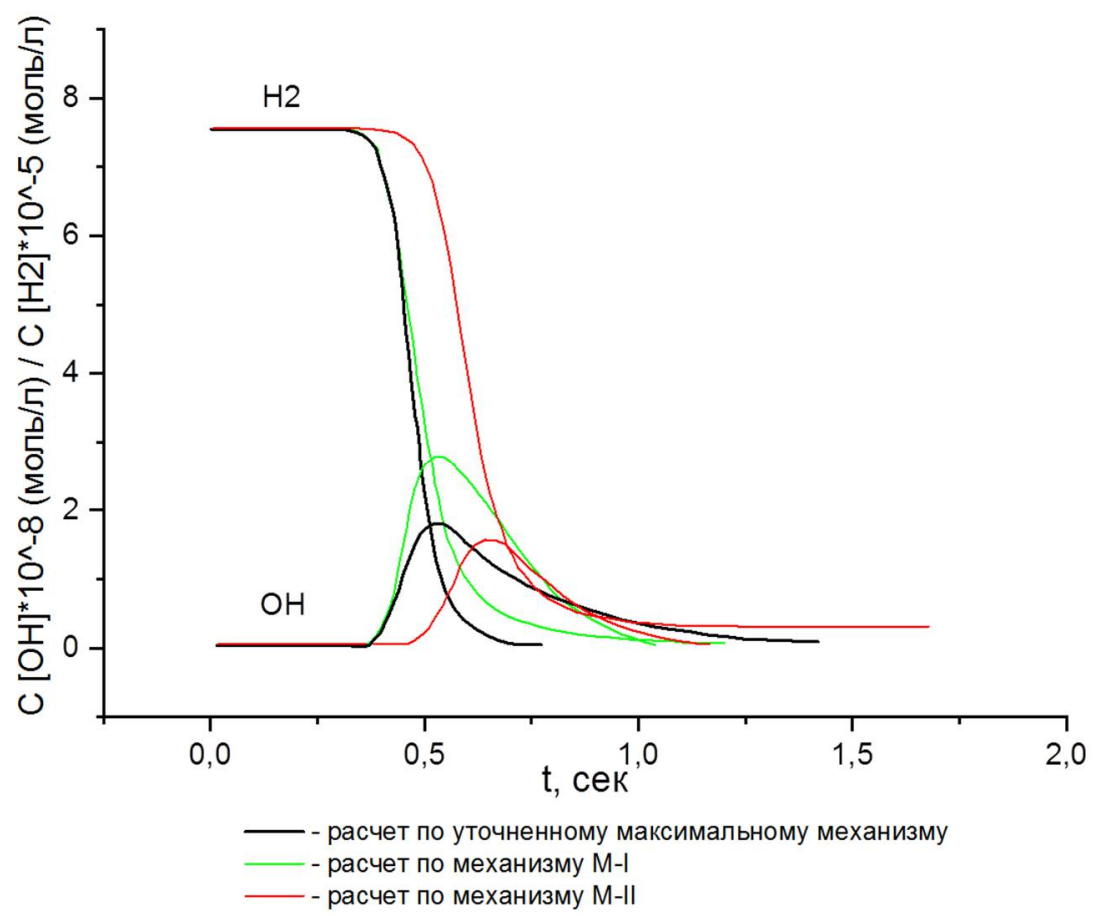

Рис. 4. Кинетические кривые концентраций $\mathrm{H}_{2}$ и $\mathrm{OH}$, стехиометрическая смесь, $430{ }^{\circ} \mathrm{C}, 5$ мм.рт.ст.

\section{3. Расчет пределов воспламенения смеси $\mathrm{H}_{2}$ и $\mathrm{O}_{2}$}

Пределы воспламенения возможно определять расчетным путем из анализа критических условий. Так, разработанный в работе [22] алгоритм позволяет получить аналитическое выражение для младшего коэффициента характеристического полинома матрицы Якоби $J$. Обращение в ноль собственного значения $J$ соответствует уравнению $C_{k}(P, T)=0$, т.е. корни этого уравнения и определяют пределы воспламенения. Главные члены этого уравнения, не зависящие от концентраций радикалов, имеют вид 


$$
C_{k}(P, T)=2 \frac{k_{2} P^{2} \alpha_{\mathrm{H}}}{k_{31}^{0}} \frac{k_{3} P^{2} \alpha_{\mathrm{O}}}{k_{32}^{0}} \frac{k_{4} P^{2} \alpha_{\mathrm{H}}}{k_{33}^{0}}-\left(\frac{k_{2} P^{2} \alpha_{H}}{k_{31}^{0}}+1\right)\left(\frac{k_{4} P^{2} \alpha_{\mathrm{H}}}{k_{33}^{0}}+1\right)\left(\frac{k_{11} P^{3} \alpha_{\mathrm{O}}}{k_{34}^{0}}+1\right)+\frac{k_{3} P^{2} \alpha_{\mathrm{O}}}{k_{32}^{0}},
$$

где $k_{2}, k_{3}, k_{4}, k_{11}$ - константы, соответствующие прямым реакциям; $k_{31}, k_{32}, k_{33}, k_{34}$ - гетерогенные константы, у которых выделен множитель $P$ полного давления $k_{g}=k_{g}^{0} / P ; \alpha_{\mathrm{H}}, \alpha_{\mathrm{O}}-$ парциальные давления водорода и кислорода.

Таким образом, условие перехода через ноль собственного значения матрицы $J$ кинетической системы, соответствующей схеме М-I, позволило рассчитать из анализа критических условий пределы воспламенения кислород-водородной смеси.

Результат вычисления пределов воспламенения по уточненному максимальному механизму и по сокращенным механизмам M-I и M-II, а также значения пределов воспламенения, полученные из анализа критических условий, приведены на рис. 5 в сравнении с экспериментальными данными Льюиса [13] и Эгертона [14]. Сокращение схемы от 34 до 12 обратимых реакций (механизм M-1) дает относительно небольшое отклонение от экспериментально установленных пределов воспламенения. Однако исключение реакций 18 и 33 (механизм МII), доли которых самые маленькие среди оставшихся, приводит к значительному отклонению верхнего предела воспламенения при давлениях выше 10 мм.рт.ст. (рис. 5, кривая 3). Исключенные реакции 18 и 33 представляют собой реакции гибели активных радикалов, что приводит к ослаблению процессов рекомбинации в механизме М-II по сравнению с максимальным, в результате с увеличением общего давления концентрации радикалов после достижения максимума остаются постоянными или очень медленно падают. Этим, видимо, и обусловлено резкое отклонение второго предела в механизме М-II от экспериментально наблюдаемого или полученного по предыдущим схемам. Таким образом, упрощенный механизм M-I может использоваться для анализа критических условий, таких как взрыв или воспламенение [21].

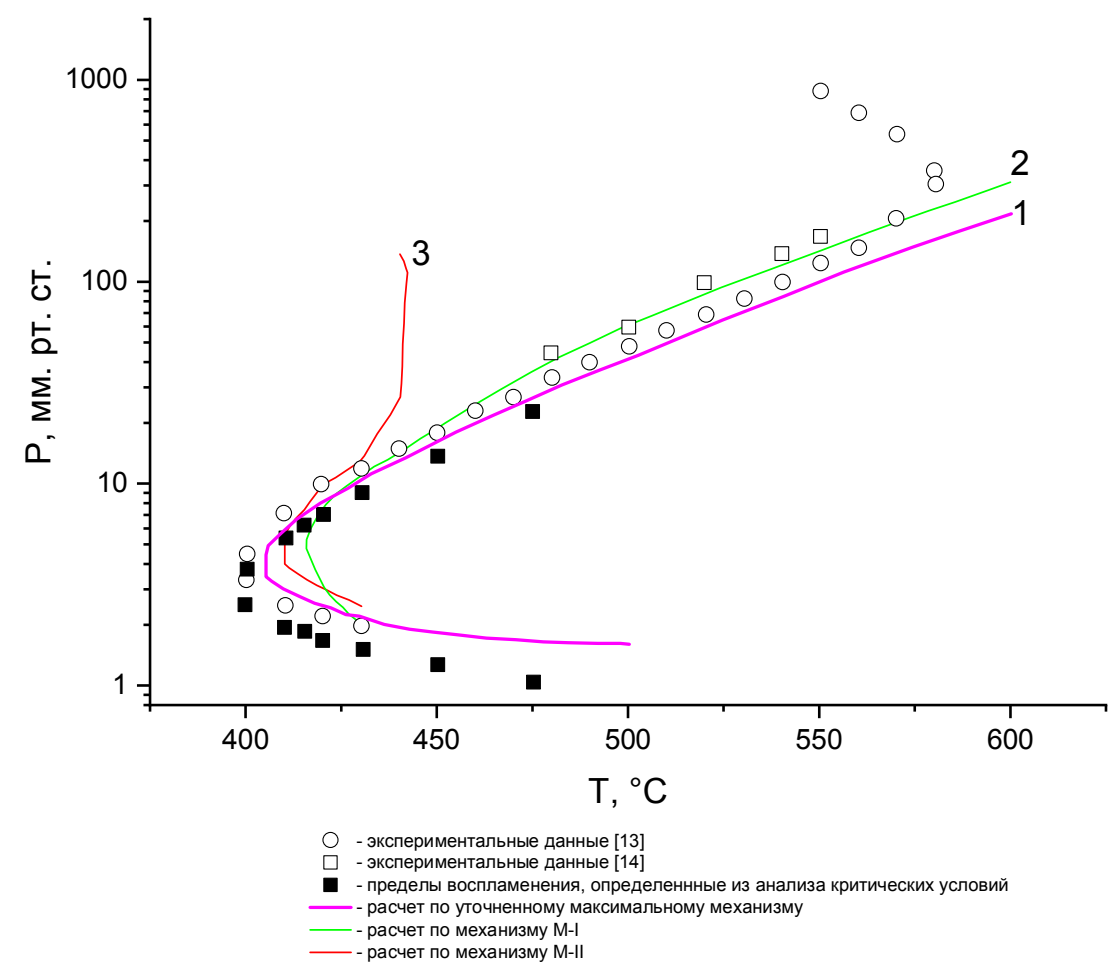

Рис. 5. Пределы воспламенения стехиометрической смеси $\mathrm{H}_{2}$ и $\mathrm{O}_{2}$ 
Полученные в данной работе параметры можно назвать взаимно согласованными, так как они описывают экспериментальные данные, в то время как ни один предлагаемый в литературе набор параметров не дает такого описания. Представляется особенно важным, что два принципиально различных эксперимента описывается одним и тем же набором констант.

\section{4. Заключение}

Для редуцирования максимального механизма горения водорода впервые предложено использовать метод термодинамического анализа, учитывающий термодинамический и кинетический факторы, а определяемая при этом термодинамическая доля характеризует значимость отдельной реакции в течение всего процесса.

Проведенный в данной работе термодинамический анализ максимальной схемы (механизма) горения водорода (включающей 68 реакций), позволил выделить 8 наиболее значимых реакций. Для этих реакций выполнен расчет кинетики горения стехиометрической смеси $\mathrm{H}_{2}+\mathrm{O}_{2}$, соответствующий экспериментальным данным [12], по результатам которого уточнены значения 8 констант. Использование уточненных (определенных) констант в максимальной схеме [16] позволяет проводить описание всех экспериментальных кинетических кривых Ковальского [12] единым набором реакций, что подтверждает надежность их определения.

В работе показано, как применение метода термодинамического анализа для уточненной максимальной схемы позволяет сократить механизм горения водорода с сохранением достаточной точности процесса горения водорода - расчет пределов воспламенения по механизму М-I в интервале давлений $1 \div 200$ мм.рт.ст. и температур $400^{\circ} \mathrm{C} \div 600^{\circ} \mathrm{C}$ хорошо совпадает с расчетом по максимальному механизму и экспериментальными данными Льюиса и Эгертона.

Полученный в работе механизм М-І позволяет достоверно описывать экспериментальные данные по кинетике горения и пределам воспламенения стехиометрической смеси $\mathrm{H}_{2}$ и $\mathrm{O}_{2}$, что может быть использовано при моделировании процессов горения водорода в различных прикладных задачах.

\section{Литература}

1. A.M. Starik, N.S. Titova, L.V. Bezgin, V.I. Kopchenov; The promotion of ignition in a supersonic $\mathrm{H}_{2}-$ air mixing layer by laser-induced excitation of $\mathrm{O}_{2}$ molecules: Numerical study // Combustion and Flame. - V. 156. - Issue 8. - 2009. - pp. 1641-1652.

2. E.W Christiansen, C.K Law, C.J Sung; Steady and pulsating propagation and extinction of rich hydrogen/air flames at elevated pressures // Combustion and Flame. - V. 124. - Issues 1-2. - 2001. - pp. 35-49.

3. P. Boivin, C. Jimenez, A. L. Sanchez, F.A. Williams; An explicit reduced mechanism for H2-air combustion // Proceedings of the Combustion Institute. - 33. - 2011. - pp. 517-523.

4. V.V. Gubernov, V. Bykov, U. Maas. Hydrogen/Air Burner-Stabilized Flames at Elevated Pressures // Combustion and Flame. - V. 185. - 2017. - pp. 44-52.

5. S. Sharath, Girimaji, Brau Carinne; Composition-Space Behavior of Diffusion-Reaction Systems // Theoretical and Computational Fluid Dynamics. - 2004. - V. 17. - Issue 3. - pp. 171-188.

6. V.V. Gubernov, A.V. Kolobov, V. Bykov, U. Maas; Investigation of Rich Hydrogen-Air Deflagrations in Models with Detailed and Reduced Kinetic Mechanisms // Combustion and Flame. - V. 168. - 2016. - pp. 32-38.

7. U. Maas, J. Waryatz; Ignition Processes in Hydrogen-Oxygen Mixtures // Combustion and Flame. 1988, - V. 74. - N 1. - pp. 53- 69.

8. U. Maas, S.B. Pope; Simplifying Chemical Kinetics: Intrinsic Low-Dimensional Manifolds in Composition Space // Comb. Flame. - 88. - 1992. - pp. 239-264. 
9. Francesca Pianosi, Keith Beven, Jim Freer, Jim W. Hall, Jonathan Rougier, David B. Stephenson, Thorsten Wagener; Sensitivity Analysis of Environmental Models: A Systematic Review with Practical Workflow // Environmental Modelling \& Software. - V. 79. - 2016. - pp. 214-232.

10. Evgeni V. Nikolaev, Jordan C. Atlas, Michael L. Shuler; Sensitivity and Control Analysis of Periodically Forced Reaction Networks Using the Green's Function Method // Journal of Theoretical Biology. - V. 247. - Issue 3. - 2007. - pp. 442-461.

11. Г.М. Писаренко, А.Г. Погорелов; Планирование кинетических исследований. - М.: Наука. 1969. - $176 \mathrm{c}$.

12. A.A. Kovalski // Phys. Z. Sow. - 1933. - V. 4. - № 5. - P. 723.

13. Б. Льюис, Г. Эльбе; Горение, пламя и взрывы в газах. - М.: Мир, 1968. - 592 с.

14. A. Egerton, D.R. Warren. Kinetics of the Hydrogen Oxygen Reaction // Proc. Roy. Soc. $-1951,-$ V. A204, - N 2. - pp. 465-476.

15. A.L. Sanchez, F.A. Williams; Recent Advances in Understanding of Flammability Characteristics of Hydrogen // Progress in Energy and Combustion Science. - 2014. - 41. - pp. 1-55.

16. Димитров В.И. Простая кинетика. - Новосибирск: Наука, 1982. - 383 с.

17. Димитров В.И., Быков В.И., Яблонский Г.С. О характеристиках сложной химической реакции. В кн. Горение и взрыв. - М.: Наука, 1977. - С. 565-570.

18. Горбань А.Н. Обход равновесия (уравнения химической кинетики и их термодинамический анализ). - Новосибирск: Наука, 1984. - 224 с.

19. Брин Э.Ф., Павлов Б.В. Применение одной модификации градиентного метода поиска экстремума для оценки кинетических параметров // Кинетика и катализ. - 1975. - Т. 16. - № 1. - С. 233-240.

20. Семенов Н.Н. О некоторых проблемах химической кинетики и реакционной способности : Избранные труды. - Т. 3. - М.: Наука, 2005. - 499 с.

21. В.В. Азатян; Цепные процессы и нестационарность состояния поверхности // Успехи химии. 1985. - T. 14. - № 1. - С. 33-60.

22. А.Н. Иванова, Б.Л. Тарнопольский; Об одном подходе к выяснению ряда качественных особенностей поведения кинетических систем и его реализация на ЭВМ // Кинетика и катализ. 1979. - Т. 20. - № 6. - С. 1541-1548. 